\title{
Polymer Characterization with the Atomic Force Microscope
}

\author{
U. Maver, T. Maver, Z. Peršin, M. Mozetič, \\ A. Vesel, M. Gaberšček and K. Stana-Kleinschek
}

Additional information is available at the end of the chapter

http://dx.doi.org/10.5772/51060

\section{Introduction}

\subsection{Atomic force microscopy}

Atomic force microscopy is a powerful characterization tool for polymer science, capable of revealing surface structures with superior spatial resolution [1]. The universal character of repulsive forces between the tip and the sample, which are employed for surface analysis in AFM, enables examination of even single polymer molecules without disturbance of their integrity [2]. Being initially developed as the analogue of scanning tunneling microscopy (STM) for the high-resolution profiling of non-conducting surfaces, AFM has developed into a multifunctional technique suitable for characterization of topography, adhesion, mechanical, and other properties on scales from tens of microns to nanometers [3].

\subsection{The technique}

A schematic representation of the basic AFM setup is shown in Figure 1. Using atomic force microscopy (AFM), a tip attached to a flexible cantilever will move across the sample surface to measure the surface morphology on the atomic scale. The forces between the tip and the sample are measured during scanning, by monitoring the deflection of the cantilever [1]. This force is a function of tip sample separation and the material properties of the tip and the sample. Further interactions arising between the tip and the sample can be used to investigate other characteristics of the sample, the tip, or the medium in-between [4].

\subsubsection{Force between the sample and the tip}

To understand the mechanisms behind the interacting components in multi-component formulations, we have to take into account all the contributing forces. This is especially 
important if a quantitative analysis of the interaction is required, like in the case of interactions between polymers and biological macromolecules [6]. The forces between the tip and the substrate have short- and long-range contributions. When measurements are performed, it is crucial that we can separate the contributions of various forces and eliminate the undesired ones. This ensures the measurement of desired sample properties only and makes further quantitative analysis possible [7]. In vacuum, chemical forces of very short range (less than $1 \mathrm{~nm}$ ), electrostatic, magnetic and Van der Waals forces can be determined, while in air forces with longer range, which can be up to $100 \mathrm{~nm}$, cover them, making the measurements mostly qualitative [8]. At room conditions water moisture can condense on the tip, which is a source of capillary force. Capillary forces are relatively big and can cover the contributions of other forces; therefore they have to be avoided if possible. The latter is possible by measuring in special, water free conditions, like in a $\mathrm{N}_{2}$ or Ar atmosphere or in liquid environments.

To represent forces on the atomic level, different potentials corresponding to changes of potential energy at various particle positions, are used. Known empirical models used to illustrate chemical bonds are the Lennard-Jones and Morse potential [9]. These models quite satisfactory fit the force regime curve shown in Figure 2, which represents the course of tipsample interaction.

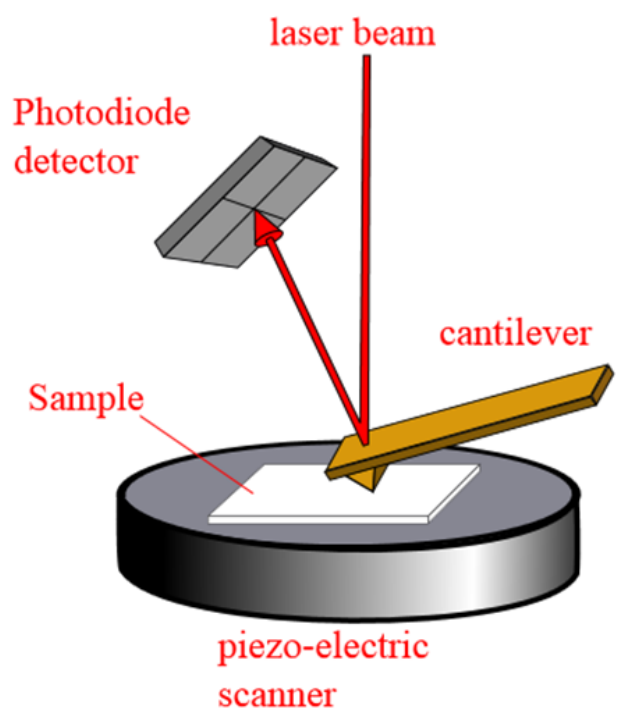

Figure 1. Schematical representation of the AFM. The image was reproduced with permission of C. Roduit [5]. 




Figure 2. Force regimes governing the AFM measurement.

\subsubsection{AFM modes for polymer examination}

Many different variations of the basic AFM setup have been developed through the years of its use. Although most of them are applicable to all types of samples, not all yield the same amount and quality results. Proper use of these versatile measurement variations enables one to study and understand processes even at the fundamental, namely molecular level [10]. Considering various different samples, several modes have been developed and adapted to cope with the demand of field specific research [11]. In the scope of the next few paragraphs only some of the most popular will be presented.

\subsubsection{Contact mode}

Contact mode was the first developed mode of atomic force microscopy. In this mode, the tip is moving across the surface and deflects according to its profile (Figure 3). Two types of contact mode measurements are known, the constant force and the constant height mode. In the constant force type, a feedback loop is used to move the sample or the tip up and down and keep its deflection constant. The value of z-movement is equal to the height changes of the sample's surface. The result of such measurement is the information about the surface topography. Since the tip is in constant contact with the surface, significant friction forces, which can destroy or sweep soft samples like polymers or biological macromolecules on the surface, appear [12].


Figure 3. Schematic representation of the contact mode. The image was reproduced with permission by C. Roduit [5]. 
The other type of contact mode AFM measurement is based on the constant height, while the forces are changing. In this case, the cantilever deflection is measured directly and the deflection force on the tip is used to calculate the distance from the surface. Since no feedback loop is required for this type of measurement, it is appropriate for quick scans of samples with small height differences (if height differences are big, the tip will very likely crash into the surface, by which it gets destroyed or damages the samples' surface). With this type of measurements atomic resolution was achieved at low temperatures and in high vacuum. Such measurements are often used for quick examination of fast changes in biological structures [13].

\subsubsection{Noncontact mode}

In noncontact mode, the sample's surface is investigated using big spring constant cantilevers. The tip attached to the cantilever is hovering very close to the surface (at a distance of approximately 5-10 nm), but never gets into contact with it, hence the name noncontact mode (Figure 4). A major advantage of this mode is negligible friction forces, making this mode capable for measurements of biological and polymeric samples without alteration of their surface. The biggest drawbacks of this mode are low lateral and zresolution when compared to the contact mode. Recently it was used for characterization of single polymer chains [14].



Figure 4. Schematical depiction of the non-contact AFM mode.

\subsubsection{Amplitude. modulation mode or dynamic force mode}

This mode is often called the intermittent-contact or tapping mode and it eliminates major weaknesses of the noncontact mode (such as the low lateral and z-resolution). Instead of hovering above the sample, the cantilever vibrates above the surface and moves through the force gradient above the surface, during which it might momentarily touch the surface [15]. Due to interactions of the AFM tip with the sample surface, the amplitude of vibrations decreases and a phase shift occurs (Figure 5). We can choose either of these parameters (amplitude or phase shift) and keep it constant through the feedback loop by moving either the sample or the tip in z-direction. This gives us information about the surface topography similar to the contact mode. To measure in the amplitude modulation mode we need much stiffer cantilevers, which exhibit the smallest possible damping factors (this factor is 
commonly referred to as the Q-factor) [16]. Amplitude modulation mode is the most often used AFM mode due to its high resolution, almost non-destructive nature of the imaging and its applicability in air and also in liquid conditions [17].



Figure 5. Schematical representation of the amplitude modulation mode. Parts of the image were reproduced with permission by C. Roduit [5].

\subsubsection{Force spectroscopy}

Force spectroscopy has proved to be one of the most promising techniques using AFM. In an AFM experiment, a tip is attached to a flexible cantilever, which is moved across the sample surface. During this procedure, the surface morphology is measured with a nanometer resolution. Upon contact with the sample surface, the tip experiences a force, which is monitored as a change in the deflection of the cantilever [18]. This force is a function of tip sample separation and the material properties of the tip and the sample and can be used to investigate other characteristics of the sample, the tip, or the medium in-between [4]. The procedure of an AFM force measurement is schematically depicted in Figure 6 and goes as follows: the tip attached to a cantilever spring is moved towards the sample in a normal direction, during this movement the vertical position of the tip and the deflection of the cantilever are recorded and converted to force-versus-distance curves, briefly called force curves [1].

In the early nineties only skilled and specialized physicists were able to interpret the complex behavior, which occurs after an AFM tip gets close to a specific sample surface. But these days many more researchers try to explore these measurements to better understand mechanisms behind more and more phenomena. In addition to evaluation of interaction forces between the tip and model surfaces, AFM can also produce two-dimensional chemical affinity maps by modifying the cantilever tip with specific molecules [19]. In such a way, it is possible to characterize differently responding regions on the material's surface, resulting in a better understanding and, consequently, application of the examined materials [20]. In this way even quantitative data can be gathered, which can be used to identify the forces involved in specific biological systems [21]. 


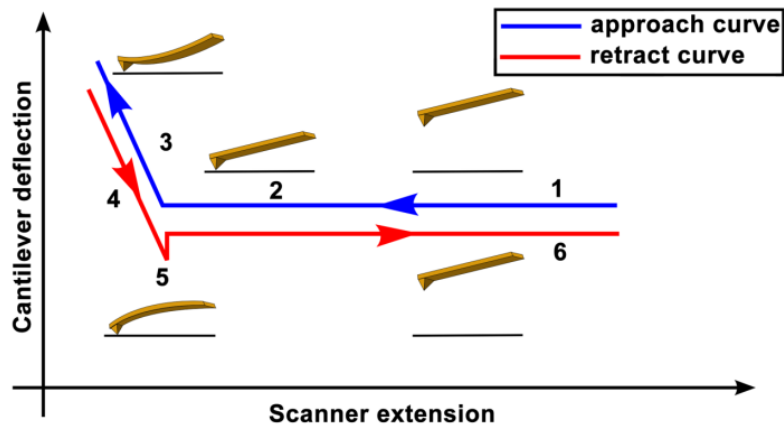

Figure 6. A typical force curve. When approaching the surface, the cantilever is in an equilibrium position (1) and the curve is flat. As the tip approaches the surface (2), the cantilever is pushed up to the surface being deflected upwards, which is seen as a sharp increase in the measured force (3). Once the tip starts retracting, the deflection starts to decrease and passes its equilibrium position at (4). As we start moving away from the surface the tip snaps in due to interaction with the surface, and the cantilever is deflected downwards (5). Once the tip-sample interactions are terminated due to increased distance, the tip snaps out, and returns to its equilibrium position (6). The image was reproduced with permission by C. Roduit [5].

Mapping chemical functional groups and examining their interactions with different materials is of significant importance for problems ranging from lubrication and adhesion, to the recognition of biological systems and pharmacy [22]. Changing environmental conditions during the measurement has also been extensively used to monitor changes in the interactions between different functional groups and surfaces to simulate the material behavior upon exposure to a real environment [23].

\section{Tip functionalization}

At the moment, one of the most promising AFM related techniques for polymer examination is surely the chemical force microscopy (CFM) $[2,24]$. CFM enables the measurement of interactions appearing between polymer molecules or polymers, and different surfaces [23]. This additional information allows the prediction of final material characteristics based on the examined polymers, even before their finalization. Quantitative assessment of the involved forces and their extent makes it easier to choose the correct polymers for achieving desired interactions between the materials used in several different interest fields (adhesion, adsorption, repelling etc.). Multilayer polymeric materials are lately also the first choice materials for the preparation of modern wound dressings. When sticking together layers of different polymeric origins, their interaction gains importance regarding the behavior of the final product. A CFM experiment has to be conducted with specially designed tips, which for themselves act as chemical sensors. Success of such measurements is impossible without proper tips, so choosing the right ones is crucial in this regard. Many commercial ones are available at the moment, but only some exhibit characteristics that allow for a simple and repeatable functionalization. Whilst the functionalization of tips may seem quite easy during the first iteration, it quickly becomes clear, that a lot of chemical skills are needed to bind the right species to the right place in the desired amount [19]. Additionally a lot of statistical evaluation is needed in order to prove and evaluate the success of any attachment [25]. 


\subsection{Polymers and AFM}

Polymers have found their way into all fields of science and industry over the last decades. Their potential applications range from binders in batteries [26] to composite materials in drug delivery [27]. Whilst the range of possible combinations between different monomers is endless, polymers found or based on natural polymers have recently become the subject of thorough research, once again [28]. Synthetic changes to their native structure make them even more appealing; especially cellulose derivatives exhibit a lot of potential for satisfying most industrial needs [29].

Within the field of polymer sciences, AFM has been used to quantify the entropic elasticity of single polymer chains[30], the elastic moduli of nanowires [31], single polymer chain elongation [32], molecular stiffness of hyperbranched macromolecules [33], friction of single polymers on surfaces [34], influence of temperature on the stability of single chain conformation [35], and surface glass transition temperature [36]. It has also been used to perform stretching experiments on single carboxy-mehtylated amylase [37], and to differentiate between sugar isomers [38].

\subsection{AFM measurements in polymer science}

Recent progress in the understanding of the underlying mechanisms during AFM force measurements enabled thorough research of the interaction between different polymer molecules and the materials, with which these get in contact upon use. Such knowledge is of utter importance in the development stages of polymeric materials, because they allow prediction of materials behavior during use. The use of controlled environments during measurement enables the simulation of the exact conditions one desires, while the measurement in liquids allows measurements in even simulated physiological conditions, which is especially desired in the testing stages of drug delivery systems.

Without proper experiment design, quantitative measurements using AFM are not possible. In this light several preparation steps have to be included in the planning phase of an experiment (Figure 7). On the following pages, we will explain them a little further and expand them with our own results and experiences.

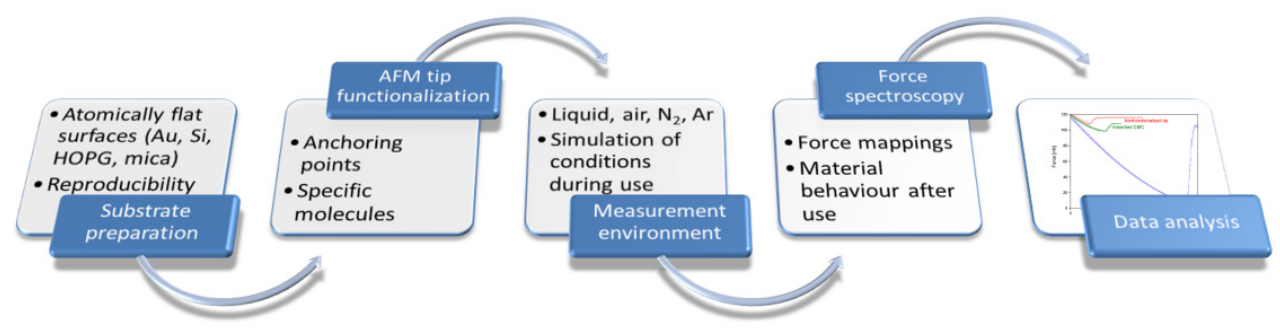

Figure 7. Schematical depiction of the necessary steps for successful AFM measurement. 


\subsubsection{Substrate preparation for AFM measurements}

Chemical force microscopy (CFM) was derived from AFM for the examination of interactions between different materials and even molecules by exploiting their chemical characteristics [39]. Quantitative assessment of such interactions can be used for identification purposes, for determination of compatibility between different materials to be put into one single final product, and to predict interactions with the target site in drug delivery systems [40].

CFM is best used with a defined experimental setup, comprising model surfaces, a controlled environment during measurements and materials of high purity. When using a high resolution technique like AFM, we have to be very careful not to confuse the information about the desired species with the substrate characteristics [41]. That is why atomically flat surfaces, apart from mica, which is commonly used for much longer, were introduced a couple of years ago, when researchers realized that not all of the data they gathered corresponded to actual species' properties, but were in fact more related to the substrates' characteristics [42]. Atomically flat surfaces are free of surface roughness and proper choice of an inert material for their preparation makes it possible to gather reliable high resolution data after desired sample attachment [43].

During our research we had to find the best possible technique to prepare such surfaces on a daily basis. Therefore we upgraded and combined different previous methods into one highly efficient preparation procedure, which enabled us to progress much faster in our experiments. A detailed explanation of this method can be found elsewhere [44], while a brief description is depicted in Figure 8 and goes as follows. Prior to any preparation steps, all used laboratory accessories were cleaned in a multi-step procedure, combining different chemicals, to assure extreme cleanliness. In the next step, high-grade mica was coated with gold of high purity. A two stage heating/annealing step was introduced afterwards, which yielded atomically flat gold terraces of sizes in the range from a couple hundred nm to 2 microns.



Figure 8. Scheme of the annealing procedure with corresponding photographs. The initial cleaning step comprises three passes of the gold coated mica piece through the hydrogen flame [44]. 
The value of such substrates cannot be evaluated without their inclusion into sample preparation. In our case, we tested them by preparing a sample with attached carbon nanotubes. If their morphology has to be evaluated, we have to use flat surfaces, which do not temper their actual properties, measured on the nanoscale. In our study, the substrates and samples were evaluated using two different types of microscopy, namely the scanning electron microscopy (SEM) and AFM. Figure 9 shows the improvement from not annealed to annealed surface with attached test molecules.

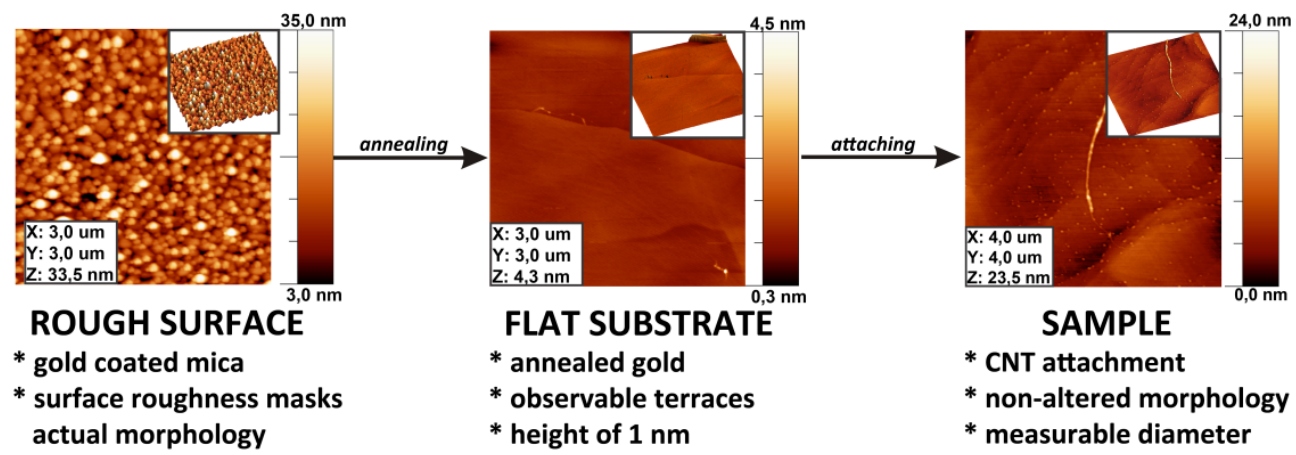

Figure 9. Progress from non-annealed gold-coated mica to the actual sample preparation and examination.

\subsubsection{AFM tip functionalization for chemical sensing}

Specific interaction mappings and identification of mechanisms behind processes require tip functionalization, which turns AFM cantilevers into chemical sensors. Depending on the degree of surface coverage with newly added functional species on the tip surface, one can measure even single molecule interactions. Such high resolution is desired, when interactions between biomolecules are tested, especially when novel drug targets are being investigated. Tip functionalization depends on the tip composition and on the desired functional groups, which in turn serve as anchoring points for more specific chemical sensing. Especially useful is CFM during the development of multilayered materials, which are commonly exploited in wound dressing preparation. The heterogeneity of the commonly used materials for this cause renders the preparation of their surfaces to stick together upon application on the wound, a tough job. CFM is capable of delivering such information in vitro. In our group, we introduced several ways of tip functionalization, which enable CFM measurements.

There are different types of commercially available AFM tips. We mostly use tips from two different groups. In the first are silicon-based tips, which can be functionalized in two step procedures. The first step involves the introduction of functional groups, which can serve as non-specific chemical sensors on their own. The second step adds specificity to them by binding desired molecules to these anchoring points, which serve as efficient sensors of desired species. In the second group are tips, which are coated with different coatings, 
which enable superior measuring capabilities, but on the other hand require different chemical means to transform them into chemical sensors. From this group, we use gold coated AFM tips the most, because of their relatively simple functionalization options, whilst bi-functional molecules, bearing on one end thiol moieties, which are known to stick to gold and on the other the desired species. Schemes of both mentioned preparation procedures are shown in Figure 10.

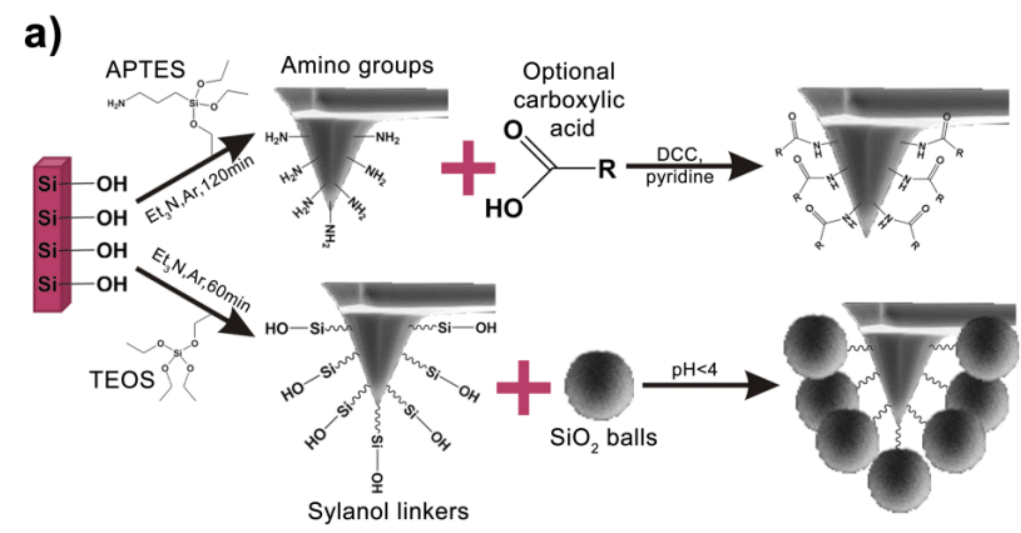

b)



Figure 10. Schematical depiction of the AFM tips functionalization procedures: a) functionalization of silicon-based tips and b) functionalization of gold-coated tips [45].

Such custom made AFM tips serve as ideal chemical sensors for many different applications [23]. As mentioned before, functionalized AFM tips can be divided into two groups, differing by the extent of their specificity towards certain chemical species. In one of our studies, we prepared functionalized AFM tips with several different functional groups [45] and showed how differently they interact with a model surface. By this, we have proven that the functionalization actually resulted in different surface functional groups and how this successful functionalization can be confirmed by using AFM. Figure 11 shows some of the results of our measurements with corresponding SEM micrographs. A clear distinction between tips with different functionalizations can be observed. 


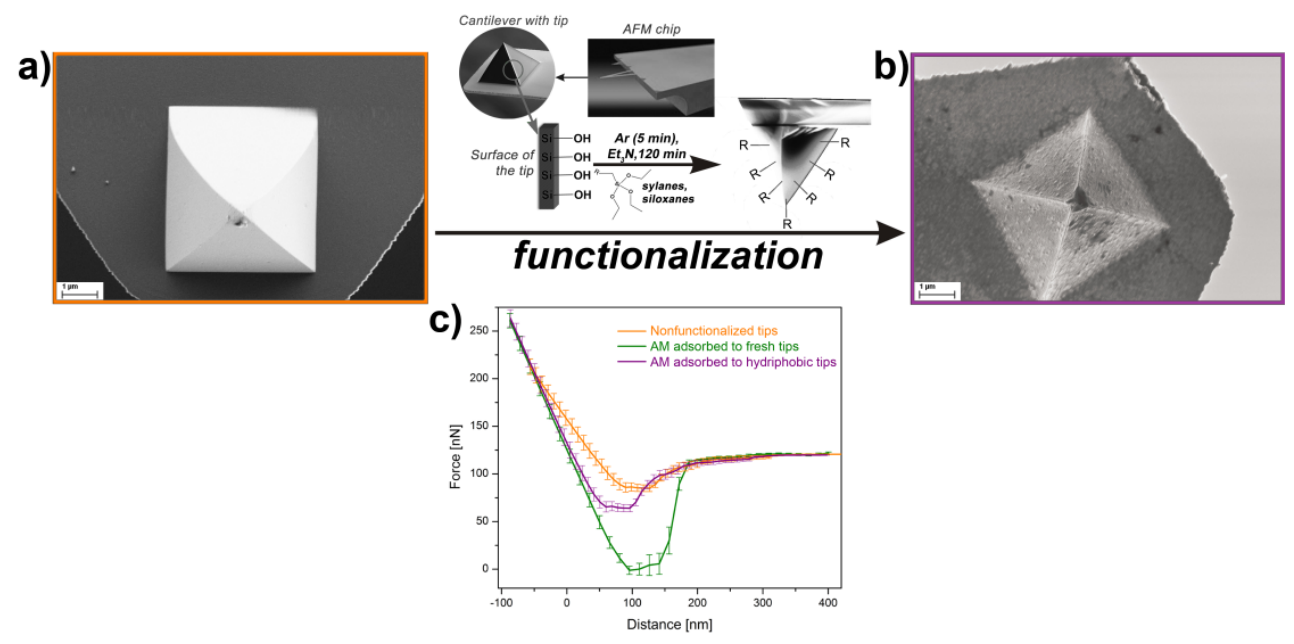

Figure 11. Scheme of the functionalization process with the corresponding results: a) SEM micrograph of nonfunctionalized AFM tips, b) SEM micrograph of an AFM tip after functionalization and c) different forces as measured with the non- and functionalized AFM tips [45].

Our results suggest that by employing some alterations to the known functionalization procedures, we are now able to attach different functional groups to the tip surface, thus providing numerous possibilities for the further attachment of a wide variety of different species. All used procedures resulted in mainly decorating the edge of tips, leaving the surroundings almost as clean as before the functionalization. In this way, there is no decrease in the response of the AFM feedback system and therefore no resolution is lost.

\subsubsection{Relationship between the polymer exposure to a specific environment and its function}

Miniaturization demands and the characteristics of specialized AFM measurements are the origin of an increasingly more and more important field of cantilever biosensing. This technique enables the determination of material and molecule behavior upon exposure to a desired environment in vitro, and by this contributes to a decrease of overall development costs for modern drug delivery systems with targeted capabilities. The main research fields, which gained the most from this technique over the past years, are pharmaceutical technology (measurements in simulated body fluids and in vitro detection of interactions between different components in complex formulations [3]), supramolecular chemistry (real time follow up of formation of self-assembled monolayers [46]), biochemistry (simulating the binding of drugs to their targets [6]), and microbiology (measurements of interactions between materials and bacteria [47]).

Our main interest in this field was the evaluation of materials performance after different exposure times in simulated physiological environments. As a consequence of the products we develop (mostly materials for use in preparation of advanced wound dressings), we tried to simplify the testing environments to simple physico-chemical 
parameters, which enable logical correlation with the results of AFM force spectroscopy [48]. Accessible in vitro testing of material response to environments similar to the ones during their use is of high importance for modern product design. Wound dressing development is not different. Several different polymer based materials are used in this field and combinations of them are often found in the most advanced products. Cellulose derivatives are by far the most spread materials for development of all kinds of plasters, bandages, gauzes etc. Because we are also focused on the development of different products made of cellulose derivatives, we tried to extend our understanding of their behavior in different environments, to better predict and more efficiently choose the right derivate for the desired purpose.

In light of the mentioned facts, we designed an experimental setup, which serves as the platform for such testing. To be reproducible, effective and to allow proper evaluation, it had to be simplified as far as possible. It consists of a model surface (atomically flat silicon wafer), two different polymer molecules (carboxy methyl cellulose and amylose) and solutions exhibiting different $\mathrm{pHs}$ and ionic strengths. The setup is schematically depicted in Figure 12.

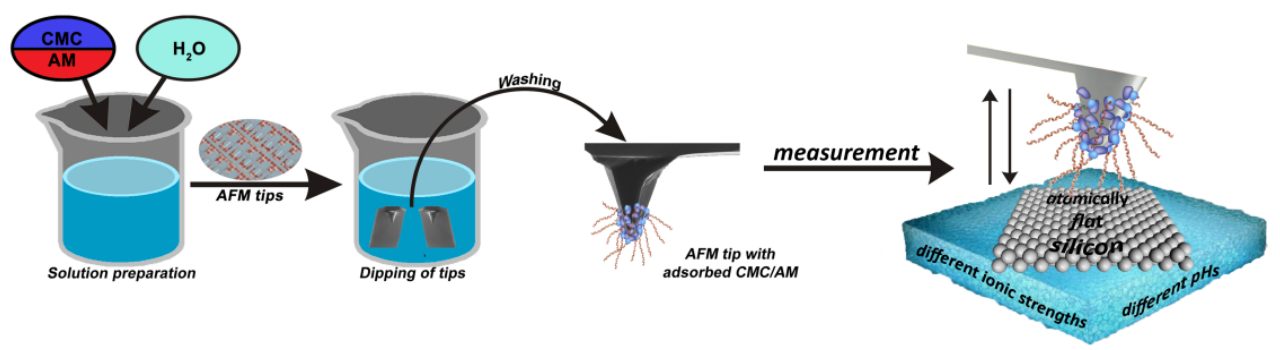

Figure 12. Scheme of the used procedure for evaluation of forces in different environments. This figure is partly reproduced from [48].

Force spectroscopy has proven to be a perfect method for assessing any interactions over a wide range of environmental conditions, especially in liquid media. The latter is especially important because capillary forces, if present (as in measurements in air), are capable of hiding smaller interaction contributions. Our research was focused on finding a reliable method for determination of environmental influences of polymer materials after exposure to a healing wound. During the healing process several physico-chemical parameters of the wound exudates change. While not all can be easily simulated, we tried to reproduce conditions, which are known to have a bigger implication on exposed materials, namely the $\mathrm{pH}$ and ionic strength. Both can induce structural changes in the polymeric chains, which in turn causes different behavior and material stability. By simplifying the setup to only two changing-parameters separately, it was possible to show that our proposed technique could serve as a good platform for assessing any changing wound-environment during healing. Some of our results are shown in Figure 13. A more detailed explanation of the measurement results can be found elsewhere [48]. 


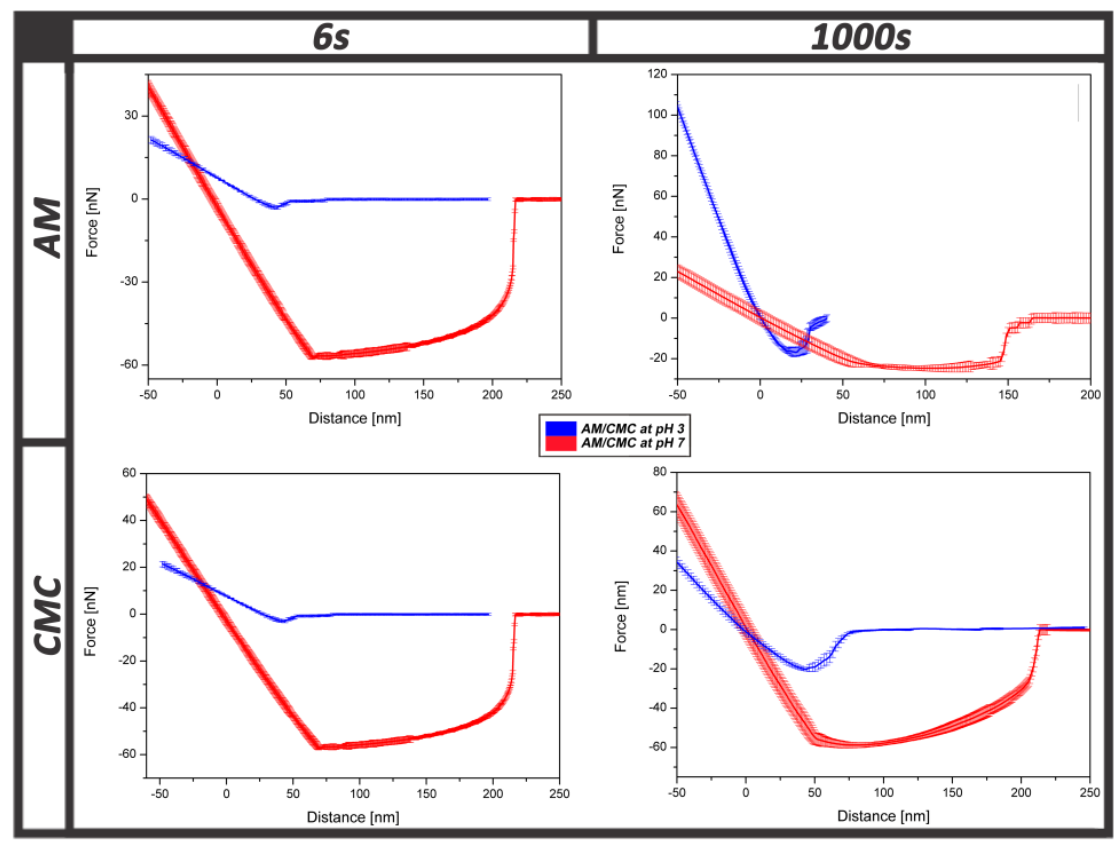

Figure 13. Force spectroscopy results for the measurements in two solutions with different pHs. TOP: retract force curves for amylose at two different measurement durations with two $\mathrm{pHs}$, BOTTOM: retract force curves for carboxymethyl cellulose at two different measurement durations with two pHs. The results are reproduced from article [48].

\subsubsection{Force spectroscopy as the source of quantitative information}

One of the greatest contributions of AFM to scientific community in the last decade is its ability to probe interaction forces between different species (surfaces, molecules, functional groups) on a quantitative basis [49]. Many researchers know that quantitative interaction mappings between species, interacting in real systems, are the basis for the comprehension of their appearance. AFM force spectroscopy yielding information about single molecules interactions was used for several important discoveries. For example, Allison et al. measured forces between adenine coated AFM tips and thymine coated surfaces, which led to the development of a methodology to study the required forces for unraveling immunoglobulin [50]. Several other research groups used the same type of experiment (attachment of specific molecules to the AFM tip edge to probe the interaction with a desired surface) to gain interaction mappings, which they used as the basis for understanding of processes on the molecular scale [51].

The mechanisms behind appearing interactions between surfaces are of utter importance for many research areas, ranging from the development of polymers for protective films to preparation of implants for medical use [52]. Quantitative assessment of these mechanisms can be used in many ways. For example, it can act as the input data for sophisticated 
modeling of polymer behavior [53], it can lead to understanding of processes on the molecular level, by which novel drugs can be developed or pathological factors filtered out several stages earlier in the development of a disease [54] or it can be used as the input data for the design of novel drug delivery systems, by which the development of such gets cheaper and less time consuming [55].

In our case, we wanted to understand a process, involved in the working process of Li-ion based battery system. Such systems comprise several components, which are connected into a sort of net via polymers, which act as the binding material [56]. Although such systems do not comprise a lot of different components, are the present ones not easy to include into calculations either due to their complex molecular structure or due to the fact that their morphology is not the same throughout the whole material. Therefore we had to develop a novel methodology of data assessment and analysis, which enables us to get more insight into the ongoing reactions during the preparation of this material [57]. The latter serves as the basis for the prediction of the loss of initial characteristics during prolonged use (the durability). By this we were able to show what binding occurs in the material, and how to correlate such data with the choice of binding material. The developed methodology is shown in Figure 14.
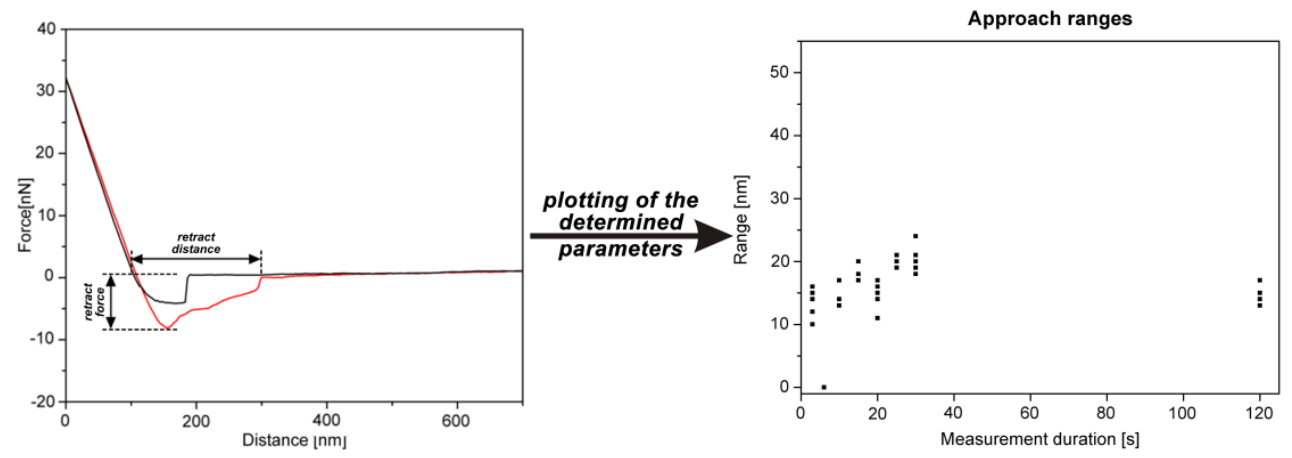

Figure 14. LEFT: typical force curve (black - approach curve, red - retract curve); RIGHT: typical plot of the extracted parameters as a function of measurement duration (approach labels were removed to increase plainness of the scheme). Reproduced with permission of the Royal Society of Chemistry from [57].

Upon introducing measurements with different durations and the final extraction of four parameters form the force curves, we were able to first define both borderline scenarios, namely the case, where a covalent bond occurs and the other, where the bond type is reversible. The next experiment was carried out at conditions, which are known to be present during the material preparation. After the comparison of this set of measured data with the previously taken ones, we found a remarkable similarity for three of the four extracted parameters. Due to the fact that the similarity was highly pronounced and due to the fact that other publications suggest the same, we are certain that the bond type in the examined material between the used binder molecules and the silicon particles is covalent. Some of the results are depicted in Figure 15, while a more detailed version can be found in our article [57]. 

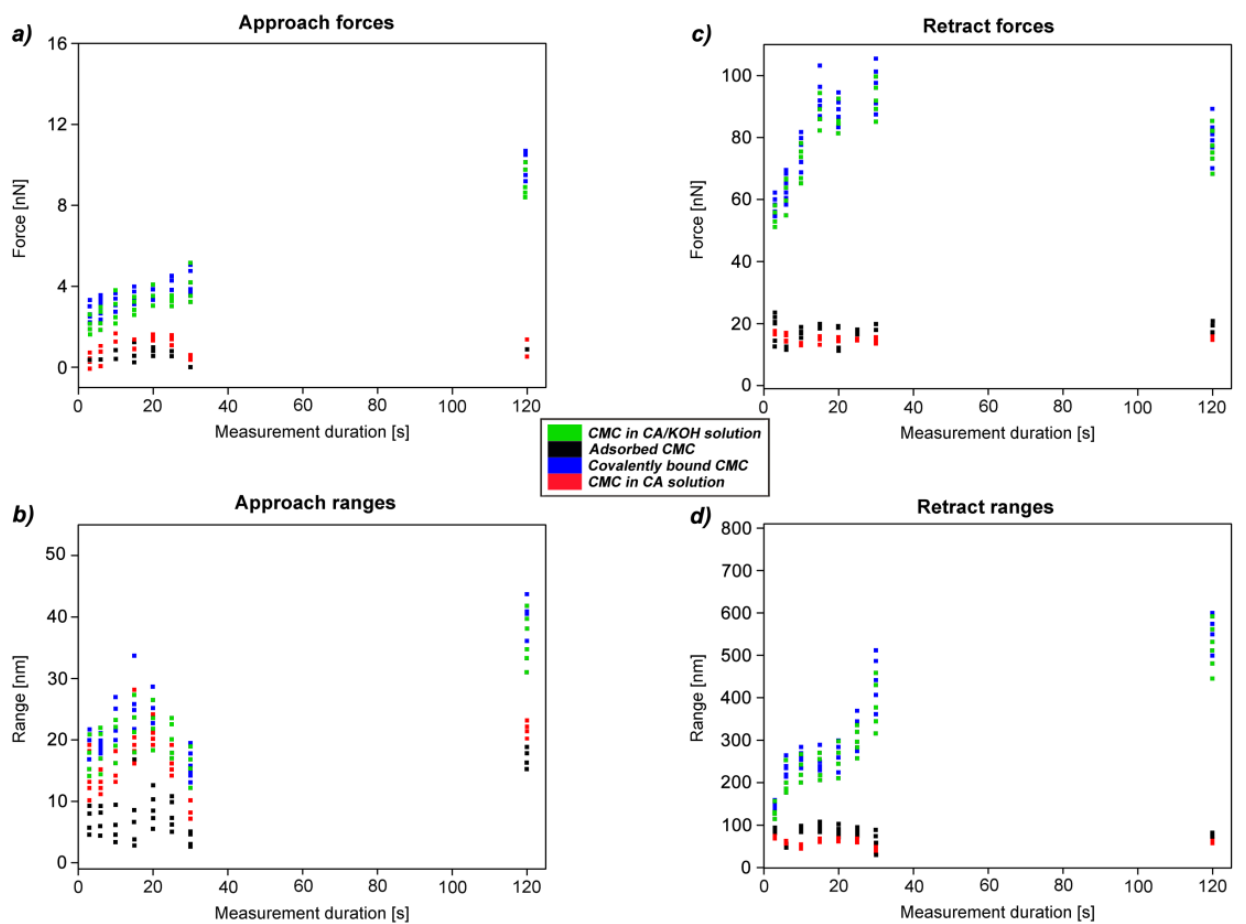

Figure 15. Plots of extracted data (determination procedure shown in Fig. 2.) for the measurements performed in water. The plots respectively show the results for four chosen parameters of each force curve taken for four different functionalization types: a) approach forces, b) approach ranges, c) retract forces and d) retract ranges vs. measurement duration. Reproduced with permission of the Royal Society of Chemistry from [57].

Such an approach is certainly not limited for the present study, but is a very good method for all other samples, where either by theory or experiment, no unambiguous data can be obtained. Additionally it can be used also for more complex molecules, where direct measurements cannot result in quantitative data or bond type confirmation. The latter is especially important in testing of polymeric materials for medical use, where the bond type between material and tissue is of high importance for the actual outcome of the healing process.

\section{Further research}

Probably the most advanced study with an AFM is the examination of single molecule behavior in its natural environment. AFM was proven as the perfect tool for identification and characterization of single polymer chains [35]. Our future efforts will be in conducting measurements on single polymer chains, compare them with results of other mechanical methods and finally try to correlate both sets of results with the final polymer material characteristics. If successful, we will be able to design and predict several novel materials, 
with a far greener and cheaper approach, which is the result of a drastically reduced number of needed experiments for desired material preparation. Our goal is to define methods, which enable effective correlation of easy obtainable laboratory data with final products characteristics even in the development stages.

\section{Conclusion}

The present chapter introduces some basic concepts of AFM measurements on polymers and explains the most used modes for their examination. Our own results are added at sections, where our knowledge represents good ground knowledge for other researchers to examine their own materials. The chapter is divided into sections, which follow the steps, needed for a thorough, and more importantly a correct analysis. Nearing the end of the chapter the complexity increases, which climaxes in the future research section, where our efforts lie at the moment.

\section{Author details}

U. Maver*

Centre of Excellence for Polymer Materials and Technologies, Ljubljana, Slovenia

National Institute of Chemistry, Ljubljana, Slovenia

T. Maver, Z. Peršin and K. Stana-Kleinschek

Centre of Excellence for Polymer Materials and Technologies, Ljubljana, Slovenia

University of Maribor, Faculty of Mechanical Engineering,

Laboratory for Characterisation and Processing of Polymers, Maribor, Slovenia

M. Mozetič

Institut "Jožef Stefan", Ljubljana, Slovenia

A. Vesel

Centre of Excellence for Polymer Materials and Technologies, Ljubljana, Slovenia

M. Gaberšček

National Institute of Chemistry, Ljubljana, Slovenia

\section{Acknowledgement}

The authors acknowledge the financial support from the Ministry of Education, Science, Culture and Sport of the Republic of Slovenia through the contract No. 3211-10-000057 (Centre of Excellence for Polymer Materials and Technologies).

\section{References}

[1] Cohen SH, Bray MT, Lightbody ML. Atomic force microscopy/scanning tunneling microscopy. New York: Plenum Press; 1994. x, 453 p. p. 
[2] Kocun M, Grandbois M, Cuccia LA. Single molecule atomic force microscopy and force spectroscopy of chitosan. Colloids and Surfaces B: Biointerfaces. 2011;82(2):470-6.

[3] Sitterberg J, Ozcetin A, Ehrhardt C, Bakowsky U. Utilising atomic force microscopy for the characterisation of nanoscale drug delivery systems. Eur J Pharm Biopharm. 2010;74(1):2-13.

[4] Butt H-J, Cappella B, Kappl M. Force measurements with the atomic force microscope: Technique, interpretation and applications. Surface Science Reports. 2005;59(1-6):1-152.

[5] Roduit C. "AFM figures", www.freesbi.ch,. Creative Commons Attribution. 2010.

[6] La R, Arnsdorf MF. Multidimensional atomic force microscopy for drug discovery: A versatile tool for defining targets, designing therapeutics and monitoring their efficacy. Life Sci. 2010;86(15-16):545-62.

[7] Uchihashi T, Higgins MJ, Yasuda S, Jarvis SP, Akita S, Nakayama Y, et al. Quantitative force measurements in liquid using frequency modulation atomic force microscopy. Appl Phys Lett. 2004;85(16):3575-7.

[8] van Noort SJT, Willemsen OH, van der Werf KO, de Grooth BG, Greve J. Mapping electrostatic forces using higher harmonics tapping mode atomic force microscopy in liquid. Langmuir. 1999;15(21):7101-7.

[9] Israelachvili JN. Intermolecular And Surface Forces: Academic Press; 2010.

[10] Cohen SH, Lightbody ML, Foundation for Advances in Medicine and Science. Atomic force microscopy/scanning tunneling microscopy 3. New York: Kluwer Academic/Plenum Publishers; 1999. viii, 210 p. p.

[11] Braga PC, Ricci D. Atomic force microscopy : biomedical methods and applications. Totowa, N.J.: Humana Press; 2004. xiv, 394 p. p.

[12] Alsteens D, Dupres V, Dague E, Verbelen C, André G, Francius G, et al. Imaging Chemical Groups and Molecular Recognition Sites on Live Cells Using AFM. In: Bhushan B, Fuchs H, editors. Applied Scanning Probe Methods XIII: Springer Berlin Heidelberg; 2009. p. 33-48.

[13] Ando T, Uchihashi T, Kodera N, Yamamoto D, Miyagi A, Taniguchi M, et al. Highspeed AFM and nano-visualization of biomolecular processes. Pflug Arch Eur J Phy. 2008;456(1):211-25.

[14] Goddard JM, Barish JA. Topographical and Chemical Characterization of Polymer Surfaces Modified by Physical and Chemical Processes. J Appl Polym Sci. 2011;120(5):2863-71.

[15] Parrat D, Sommer F, Solleti JM, Due TM. Imaging Modes in Atomic-Force Microscopy. J Trace Microprobe T. 1995;13(3):343-52.

[16] Basdogan C, Varol A, Gunev I, Orun B. Numerical simulation of nano scanning in intermittent-contact mode AFM under Q control. Nanotechnology. 2008;19(7).

[17] Nnebe I, Schneider JW. Characterization of distance-dependent damping in tappingmode atomic force microscopy force measurements in liquid. Langmuir. 2004;20(8):3195-201.

[18] Binnig G, Quate CF, Gerber C. Atomic Force Microscope. Physical Review Letters. 1986;56(9):930. 
[19] Ebner A, Wildling L, Zhu R, Rankl C, Haselgrübler T, Hinterdorfer P, et al. Functionalization of Probe Tips and Supports for Single-Molecule Recognition Force Microscopy. In: Samorì P, editor. STM and AFM Studies on (Bio)molecular Systems: Unravelling the Nanoworld: Springer Berlin / Heidelberg; 2008. p. 29-76.

[20] Kienberger F, Pastushenko VP, Kada G, Puntheeranurak T, Chtcheglova L, Riethmueller C, et al., editors. Improving the contrast of topographical AFM images by a simple averaging filter. 2006.

[21] Selvin PR, Ha T. Single-molecule techniques : a laboratory manual. Cold Spring Harbor, N.Y.: Cold Spring Harbor Laboratory Press; 2008. vii, 507 p. p.

[22] Frisbie CD, Rozsnyai LF, Noy A, Wrighton MS, Lieber CM. Functional-Group Imaging By Chemical Force Microscopy. Science. 1994;265(5181):2071-4.

[23] Kienberger F, Ebner A, Gruber HJ, Hinterdorfer P. Molecular recognition imaging and force spectroscopy of single biomolecules. Accounts Chem Res. 2006;39(1):29-36.

[24] Ito $T$, Ibrahim $S$, Grabowska I. Chemical-force microscopy for materials characterization. TrAC Trends in Analytical Chemistry. 2010;29(3):225-33.

[25] Hinterdorfer P, Gruber HJ, Kienberger F, Kada G, Riener C, Borken C, et al. Surface attachment of ligands and receptors for molecular recognition force microscopy. Colloid Surface B. 2002;23(2-3):115-23.

[26] Kaneko M, Nakayama M, Wakihara M. Lithium-ion conduction in elastomeric binder in Li-ion batteries. Journal of Solid State Electrochemistry. 2007;11(8):1071-6.

[27] Satarkar NS, Biswal D, Hilt JZ. Hydrogel nanocomposites: a review of applications as remote controlled biomaterials. Soft Matter. 2010;6(11):2364-71.

[28] Huang S, Fu X. Naturally derived materials-based cell and drug delivery systems in skin regeneration. J Control Release. 2010;142(2):149-59.

[29] Liu ZH, Jiao YP, Wang YF, Zhou CR, Zhang ZY. Polysaccharides-based nanoparticles as drug delivery systems. Advanced Drug Delivery Reviews. 2008;60(15):1650-62.

[30] Ortiz C, Hadziioannou G. Entropic elasticity of single polymer chains of poly(methacrylic acid) measured by atomic force microscopy. Macromolecules. 1999;32(3):780-7.

[31] Shanmugham S, Jeong JW, Alkhateeb A, Aston DE. Polymer nanowire elastic moduli measured with digital pulsed force mode AFM. Langmuir. 2005;21(22):10214-8.

[32] Bemis JE, Akhremitchev BB, Walker GC. Single polymer chain elongation by atomic force microscopy. Langmuir. 1999;15(8):2799-805.

[33] Shulha H, Zhai XW, Tsukruk VV. Molecular stiffness of individual hyperbranched macromolecules at solid surfaces. Macromolecules. 2003;36(8):2825-31.

[34] Kuhner F, Erdmann M, Sonnenberg L, Serr A, Morfill J, Gaub HE. Friction of single polymers at surfaces. Langmuir. 2006;22(26):11180-6.

[35] Giannotti MI, Rinaudo M, Vancso GJ. Force spectroscopy of hyaluronan by atomic force microscopy: From hydrogen-bonded networks toward single-chain behavior. Biomacromolecules. 2007;8(9):2648-52.

[36] Bliznyuk VN, Assender HE, Briggs GAD. Surface Glass Transition Temperature of Amorphous Polymers. A New Insight with SFM. Macromolecules. 2002;35(17):6613-22. 
[37] Lu Z, Nowak W, Lee G, Marszalek PE, Yang W. Elastic Properties of Single Amylose Chains in Water: A Quantum Mechanical and AFM Study. J Am Chem Soc. 2004;126(29):9033-41.

[38] Zhang Q, Marszalek PE. Identification of Sugar Isomers by Single-Molecule Force Spectroscopy. J Am Chem Soc. 2006;128(17):5596-7.

[39] Noy A, Frisbie CD, Rozsnyai LF, Wrighton MS, Lieber CM. Chemical Force Microscopy - Exploiting Chemically-Modified Tips To Quantify Adhesion, Friction, And Functional-Group Distributions In Molecular Assemblies. J Am Chem Soc. 1995;117(30):7943-51.

[40] Tumer YTA, Roberts CJ, Davies MC. Scanning probe microscopy in the field of drug delivery. Advanced Drug Delivery Reviews. 2007;59(14):1453-73.

[41] El Kirat K, Burton I, Dupres V, Dufrene YF. Sample preparation procedures for biological atomic force microscopy. J Microsc-Oxford. 2005;218:199-207.

[42] Hegner M, Wagner P, Semenza G. Ultralarge Atomically Flat Template-Stripped Au Surfaces For Scanning Probe Microscopy. Surf Sci. 1993;291(1-2):39-46.

[43] Stroh C, Wang H, Bash R, Ashcroft B, Nelson J, Gruber H, et al. Single-molecule recognition imaging-microscopy. P Natl Acad Sci USA. 2004;101(34):12503-7.

[44] Maver U, Planinšek O, Jamnik J, Hassanien AI, Gaberšček M. Preparation of atomically flat gold substrates for AFM measurements. Acta Chimica Slovenica. 2012;59(1):212-9.

[45] Maver T, Stana-Kleinschek K, Peršin Z, Maver U. Functionalization of AFM tips for use in force spectroscopy between polymers and model surfaces. Mater Tehnol. 2011;45(3):205-11.

[46] Kluge D, Abraham F, Schmidt S, Schmidt H-W, Fery A. Nanomechanical Properties of Supramolecular Self-Assembled Whiskers Determined by AFM Force Mapping. Langmuir. 2010;26(5):3020-3.

[47] Ivanova EP. Nanosacale structure and properties of microbial cell surfaces. Hauppauge, N.Y.: Nova Science Publishers; 2007. xiv, 269 p. p.

[48] Maver U, Maver T, Znidarsic A, Persin Z, Gaberscek M, Stana-Kleinschek K. Use of Afm Force Spectroscopy for Assessment of Polymer Response to Conditions Similar to the Wound, during Healing. Mater Tehnol. 2011;45(3):259-63.

[49] Roberson ED. Alzheimer's disease and frontotemporal dementia : methods and protocols. New York: Humana Press; 2011. x, 277 p. p.

[50] Allison DP, Hinterdorfer P, Han WH. Biomolecular force measurements and the atomic force microscope. Current Opinion in Biotechnology. 2002;13(1):47-51.

[51] Ebner A, Wildling L, Kamruzzahan ASM, Rankl C, Wruss J, Hahn CD, et al. A new, simple method for linking of antibodies to atomic force microscopy tips. Bioconjugate Chem. 2007;18(4):1176-84.

[52] Lehnert M, Gorbahn M, Rosin C, Klein M, Koper I, Al-Nawas B, et al. Adsorption and Conformation Behavior of Biotinylated Fibronectin on Streptavidin-Modified TiOX Surfaces Studied by SPR and AFM. Langmuir. 2011;27(12):7743-51.

[53] Junker JP, Rief M. Single-molecule force spectroscopy distinguishes target binding modes of calmodulin. Proceedings of the National Academy of Sciences. 2009. 
[54] Stolz M, Gottardi R, Raiteri R, Miot S, Martin I, Imer R, et al. Early detection of aging cartilage and osteoarthritis in mice and patient samples using atomic force microscopy. Nature Nanotechnology. 2009;4(3):186-92.

[55] Lyon LA, South AB. Direct Observation of Microgel Erosion via in-Liquid Atomic Force Microscopy. Chem Mater. 2010;22(10):3300-6.

[56] Magasinski A, Zdyrko B, Kovalenko I, Hertzberg B, Burtovyy R, Huebner CF, et al. Toward Efficient Binders for Li-Ion Battery Si-Based Anodes: Polyacrylic Acid. Acs Appl Mater Inter. 2010;2(11):3004-10.

[57] Maver U, Žnidaršič A, Gaberšček M. An attempt to use atomic force microscopy for determination of bond type in lithium battery electrodes. J Mater Chem. 2011;21(12):4071-5. 